\title{
HER-2-neu, CEA Peptides, GM-CSF, Montanide ISA-51 Vaccine
}

National Cancer Institute

\section{Source}

National Cancer Institute. HER-2-neu, CEA Peptides, GM-CSF, Montanide ISA-51 Vaccine.

NCI Thesaurus. Code C48392.

A vaccine comprised of HER-2-neu and carcinoembryonic antigen synthetic (CEA) peptides, combined with the adjuvants granulocyte-macrophage colony stimulating factor (GM-CSF), and Montanide ISA-51 with potential antineoplastic activity. HER-2-neu, CEA peptides, GM-CSF, Montanide ISA-51 vaccine may stimulate a cytotoxic T-cell response against HER-2-neu- and CEA-expressing tumor cells. The GM-CSF adjuvant stimulates the proliferation of monocytes and monocyte differentiation into macrophages and dendritic cells, immunohematopoietic elements with important antitumoral functions. Montanide ISA-51, also known as incomplete Freund's adjuvant or IFA, is a stabilized water-in-oil emulsion adjuvant containing mineral oil with mannide oleate added as a surfactant that non-specifically stimulates cell-mediated immune responses to antigens. 